Tarifverhandlungen im öffentlichen Dienst

\section{Für die „Held*innen des Alltags“}

Tarifverhandlungen im öffentlichen Dienst sind immer etwas Besonderes. Anders als in der Privatwirtschaft gibt es keinen unmittelbaren Verteilungskonflikt zwischen Unternehmen und ihren Beschäftigten. Es geht vielmehr um die Verteilung von öffentlichen Ressourcen und die sind bekanntlich meistens knapp. Allerdings entscheidet der Staat mittels Abgaben und Steuern selbst zu einem gewichtigen Anteil über die zur Verfügung stehenden Ressourcen. Damit kehrt der Verteilungskonflikt durch die Hintertür auch in die Tarifauseinandersetzungen zurück.

Würden sich die Tarifverhandlungen im öffentlichen Dienst einfach nur nach der aktuellen Kassenlage richten, so hätten die Beschäftigten in der Vergangenheit vielfach überhaupt keine Lohnerhöhungen erhalten dürfen. Tatsächlich ist die Lohnentwicklung im öffentlichen Dienst über lange Zeit hinweg deutlich hinter der Entwicklung in anderen Branchen zurückgeblieben. Erst in den letzten Jahren haben gute Tarifabschlüsse die Lücke wieder etwas verkleinern können, ohne sie freilich vollständig zu schließen. Nach wie vor verdienen Pflegekräfte, Erzieher*innen, Busfahrer*innen, Verwaltungsangestellte usw. im Schnitt deutlich weniger als viele ihre Kolleg*innen in den Industrieunternehmen.

Das Ergebnis dieser Entwicklung zeigt sich mittlerweile in einem erheblichen Personal- und Fachkräftemangel. Nach Umfragen des Deutschen Beamtenbundes fehlen jetzt schon etwa 300.000 Beschäftigte im öffentlichen Dienst. Besonders offensichtlich ist dies im Bereich der Pflege. Aber auch in den Kitas, Schulen und Berufsschulen, in der Sozialen Arbeit, bei den Sicherheitsbehörden, in Justiz und Finanzämtern, im Gesundheitsdienst, im technischen Dienst, bei den Bürgerämtern, im öffentlichen Nahverkehr werden zunehmend personelle Engpässe sichtbar. Hinzu kommt, dass der öffentliche Dienst in den nächsten Jahren eine gewaltige Pensionswelle erleben wird, die die Personalprobleme weiter verschärft. Eine Anstellung im öffentlichen Dienst ist dabei heute alles andere als ein Traumjob. Nicht nur die Bezahlung hinkt der Privatwirtschaft oft hinterher. Auch die viel gerühmte Beschäftigungssicherheit hat angesichts der Tatsache, dass im öffentlichen Dienst mehr als jeder Zweite erst einmal befristet eingestellt wird, erheblich an Ausstrahlung

(C) Der/die Autor(en) 2020. Open Access: Dieser Artikel wird unter der Creative Commons Namensnennung 4.0 International Lizenz (https:// creativecommons.org/licenses/by/4.0/deed.de) veröffentlicht.

Open Access wird durch die ZBW - Leibniz-Informationszentrum Wirtschaft gefördert. verloren. Schließlich haben Sparzwang und Personalmangel dazu geführt, dass im öffentlichen Dienst heute die Beschäftigten deutlich häufiger über Stress, Arbeitsverdichtungen und psychische Belastungen klagen, als in anderen Wirtschaftsbereichen. Ohne attraktivere Arbeitsbedingungen werden die Personalprobleme nicht zu lösen sein. Und ohne mehr Personal droht die Leistungsfähigkeit des Staates zukünftig in vielen Bereichen deutlich eingeschränkt zu werden.

An all diesen Zusammenhängen hat die Corona-Krise zunächst nichts grundlegend verändert. Die Pandemie hat im Gegenteil noch einmal allen vor Augen geführt, wie wichtig die Existenz einer funktionierenden öffentlichen Daseinsvorsorge ist. Zu Recht wurden viele Beschäftigtengruppen als „Held*innen des Alltags“ beklatscht und erwarten nun auch eine entsprechende materielle Anerkennung. In der aktuellen Tarifrunde des öffentlichen Dienstes geht es jedoch nicht nur darum, die Leistungen einiger in der Corona-Krise besonders belasteter Beschäftigungsgruppen zu honorieren. Noch wichtiger ist vielmehr ein Zeichen dafür, dass der öffentliche Dienst als Ganzes weiter aufgewertet und damit auch besser entlohnt werden muss. Denn auch nach der Corona-Krise werden die „Held*innen des Alltags" weiter gebraucht. In manchen Bereichen, wie z. B. dem öffentlichen Nahverkehr, müssen es sogar deutlich mehr werden - auch um die so dringend erforderliche Verkehrswende voranzubringen.

In der letzten Tarifrunde 2018 haben sich die Tarifvertragsparteien mit einem guten Abschluss für die Beschäftigten bei Bund und Kommunen auf den Weg gemacht, den öffentlichen Dienst insgesamt wieder attraktiver zu gestalten. Dieser Weg wurde 2019 mit einem entsprechenden Abschluss bei den Ländern fortgesetzt. Es wäre fatal, diesen Weg jetzt einfach wieder abzubrechen. Die Gewerkschaften haben mit einer Lohnerhöhung von 4,8\% eine vergleichsweise moderate Forderung aufgestellt, die unter normalen Umständen in einen noch moderateren Abschluss münden würde. Damit haben sie den erheblichen Einnahmeverlusten der öffentlichen Hand durch die Corona-Krise Rechnung getragen. Was sie nicht akzeptierten können ist hingegen, dass der Staat auf der einen Seite Milliarden Euro in die Hand nimmt, um Unternehmen $z u$ retten, bei seinen eigenen Beschäftigten aber plötzlich wieder das alte Lied der leeren Kassen singt. Spätestens an diesem Punkt ist der klassische Verteilungskonflikt in die Tarifrunde zurückgekehrt und es geht um die Frage, wer am Ende die Kosten der Krise trägt.

Thorsten Schulten

WSI - Wirtschafts- und Sozialwissenschaftliches Institut der Hans-Böckler-Stiftung thorsten-schulten@boeckler.de 